\title{
Identification of neurons responsible for feeding behavior in the Drosophila brain
}

\author{
SUN Fei ${ }^{1,2}$, WANG YiJin ${ }^{1}$, ZHOU YanQiong ${ }^{1,3}$, VAN SWINDEREN Bruno ${ }^{3}$, \\ GONG ZheFeng ${ }^{1,4} \&$ LIU Li ${ }^{1,5^{*}}$ \\ ${ }^{1}$ Institute of Biophysics, Chinese Academy of Sciences, Beijing 100101, China; \\ ${ }^{2}$ University of Chinese Academy of Sciences, Beijing 100039, China; \\ ${ }^{3}$ Queensland Brain Institute, The University of Queensland, Brisbane, Queensland 4072, Australia; \\ ${ }^{4}$ School of Medicine, Zhejiang University, Hangzhou 310058, China; \\ ${ }^{5}$ Key Laboratory of Mental Health, Chinese Academy of Sciences, Beijing 100101, China
}

Received January 15, 2014; accepted February 24, 2014; published online March 19, 2014

\begin{abstract}
Drosophila melanogaster feeds mainly on rotten fruits, which contain many kinds of sugar. Thus, the sense of sweet taste has evolved to serve as a dominant regulator and driver of feeding behavior. Although several sugar receptors have been described, it remains poorly understood how the sensory input is transformed into an appetitive behavior. Here, we used a neural silencing approach to screen brain circuits, and identified neurons labeled by three Gal4 lines that modulate Drosophila feeding behavior. These three Gal4 lines labeled neurons mainly in the suboesophageal ganglia (SOG), which is considered to be the fly's primary taste center. When we blocked the activity of these neurons, flies decreased their sugar consumption significantly. In contrast, activation of these neurons resulted in enhanced feeding behavior and increased food consumption not only towards sugar, but to an array of food sources. Moreover, upon neuronal activation, the flies demonstrated feeding behavior even in the absence of food, which suggests that neuronal activation can replace food as a stimulus for feeding behavior. These findings indicate that these Gal4-labeled neurons, which function downstream of sensory neurons and regulate feeding behavior towards different food sources is necessary in Drosophila feeding control.
\end{abstract}

feeding behavior, sugar-sensing neurons, SOG, CAFE assay, proboscis extension response (PER)

Citation: $\quad$ Sun F, Wang YJ, Zhou YQ, van Swinderen B, Gong ZF, Liu L. Identification of neurons responsible for feeding behavior in the Drosophila brain. Sci China Life Sci, 2014, 57: 391-402, doi: 10.1007/s11427-014-4641-2

Feeding is one of the most conserved innate activities of animals. How an animal decides what behaviors to engage in remains a major question in neurobiology, and feeding behavior provides an easily measured output for dissecting decision-making processes. Although diverse feeding habits have evolved to accommodate a complex array of food sources, animals, from flies to humans, react to taste molecules in a similar way: most are attracted to sugars and repelled by bitter and toxic compounds [1-3]. In addition to

*Corresponding author (email: liuli@ sun5.ibp.ac.cn) this similarity with mammals, the powerful genetic tools and many conserved metabolic elements [4,5] make Drosophila melanogaster a good model system to study feeding behavior [6-8]. In Drosophila, feeding begins with the detection of a palatable food source by the taste system. Taste perception allows flies to discriminate sweet or nutritionally rewarding food from food that is potentially contaminated or toxic, which typically tastes bitter [9-12]. Hence, the ability to taste sweetness is fundamental to survival.

The gustatory system plays a central role in the food evaluation process. Unlike humans, fruit fly taste perception 
is not restricted to one tissue. Flies sample their local chemical environment through sensilla, which are hair-like structures distributed across the proboscis (labella), internal mouthpart organs, legs, wings, and ovipositor [13-15]. On the proboscis, taste sensilla have a pore at their tip that allows chemicals to interact with gustatory receptor neurons (GRNs) [16], which reside underneath the sensilla and send axons directly to the central nervous system $[2,17,18]$. Taste detection is mediated by distinct sets of gustatory receptors, encoded by approximately 60 gustatory receptor (Gr) genes [19-22]. Multiple Gr genes are expressed in each GRN, which in turn can be divided into different subtypes according to certain features related to responsiveness [18,23]. For example, eight Grs (Gr5a, Gr61a and Gr64a-Gr64f) are partially co-expressed in a single GRN, which are characterized as sugar-sensing GRNs because the expressing receptors are responsive to sugars [24-26]. Specifically, in sugar-sensing GRNs, Gr5a is necessary for the response to a small subset of sugars, including trehalose [24,27,28]. In contrast, Gr64a is essential for the detection of multiple other sugars, including sucrose, glucose, and maltose $[24,26,29]$. More recent studies demonstrate that Gr64e is a receptor for glyce- rol [30] and Gr64f, as a co-receptor, functions with Gr5a and Gr64a to detect most sugars [25].

Feeding behaviors are highly regulated not only by the peripheral sensory system but also by neurons in the brain. For example, motor neurons drive proboscis extension and fluid ingestion [31,32]. A set of dopaminergic neurons classified as ventral unpaired medial (TH-VUM) neurons mediates increased sugar sensitivity during hunger [33,34]. In addition, various neuropeptide (Hugin, NPF, DILP, etc.)expressing neurons modulate the initiation/termination of a meal and interact with other sensory systems to alter food odor attractiveness (reviewed in [35]). Despite a growing body of knowledge regarding feeding behavior regulation in flies, much less is known about neural processing underlying appetitive behavior. It is thought that peripheral attractants and internal metabolic cues are integrated in the brain before being output to shape feeding behavior [36].

In this study, to dissect the neural circuits involved in feeding control and to identify previously uncharacterized neurons involved in the process, we conducted a behavioral screen to identify sugar-responding neurons in the central nervous system. We identified three Gal4 lines that label groups of neurons in the central nervous system responsible for food intake. The neural activity of these neurons is necessary to generate normal feeding behavior.

\section{Materials and methods}

\subsection{Experimental animals}

Drosophila stocks were maintained on standard medium with a 12:12 h light/dark cycle [37]. Flies not expressing heat-shock inducible constructs were raised and tested at $25^{\circ} \mathrm{C}$. Flies expressing UAS-dTrpA1 were raised at $18^{\circ} \mathrm{C}$ and heat-shock was performed by infrared laser or by raising the room temperature to $30^{\circ} \mathrm{C}$ during tests.

All the flies for behavioral experiments were 3-7-dayold females. Flies were first collected and fed on regular food for $1 \mathrm{~d}$ and then starved with access to water for $22 \mathrm{~h}$. All the behavioral experiments were carried out between 10:00 a.m. to 2:00 p.m. to control for circadian influences.

The following flies were used: $\operatorname{poxn}^{70}$ [38], poxn ${ }^{4 M 22}$ [39], UAS-dORK [40], Gr5a-Gal4, Gr61a-Gal4, Gr64a-Gal4, Gr64c-Gal4, Gr64d-Gal4, Gr64e-Gal4, Gr64f-Gal4 [41], UAS-dTrpA1 [42], UAS-mCD8::GFP [43], NP115-Gal4, NP883-Gal4, NP1076-Gal4 (Drosophila Genetic Resource Center).

\subsection{CAFE assay}

The capillary feeding (CAFE) assay was carried out as described by Ja et al. [44], with the following modifications. The apparatus consisted of an empty vial capped with a cotton plug. Two capillaries (length $100 \mathrm{~mm}$, inner diameter $0.4 \mathrm{~mm}$ ) were inserted into the plug via adaptors made of truncated pipette tips. Capillaries were filled with two types of liquid food by capillary action. To reduce evaporation, experiments were carried out in a small room in which the relative humidity was kept at $80 \%$. Ten female flies were transferred to each vial by brief $\mathrm{CO}_{2}$ anesthetization and allowed to feed on the provided food sources for $3 \mathrm{~h}$. Five control vials without flies were used to determine the effects of evaporation in each experiment. Since the diameter of the capillary was constant, we measured the length of the meniscus descent to determine the evaporation rate. The length of meniscus descent in vials with flies minus the length of meniscus descent in vials without flies was calculated as food consumption. For single-type food experiments where two capillaries were filled with same liquid food, all the experimental procedures were the same as described above except that the food consumption of the two capillaries in each vial was summed to get total consumption.

\subsection{Proboscis extension reflex (PER)}

Flies were immobilized on slides in a humidified chamber for $2 \mathrm{~h}$ at $18^{\circ} \mathrm{C}$. Experiments were carried out at room temperature $\left(<25^{\circ} \mathrm{C}\right)$. Flies were first fed with water to satiation then heat shocked with an infrared laser [45] aimed at the head for $5 \mathrm{~s}$. Each fly was stimulated by heat three times, with water administered between each heat treatment, and the number of proboscis extensions was recorded. Three batches of 20-30 flies were tested for each genotype.

\subsection{Immunohistochemistry}

Three- to five-day-old female flies were collected and brain dissection was performed in cold phosphate-buffered saline 
(PBS). The samples were fixed in freshly prepared paraformaldehyde (4\% in PBS) for $3 \mathrm{~h}$ on ice, rinsed $3 \times 15 \mathrm{~min}$ in PBT (PBS with $0.5 \%$ Triton X-100), followed by blocking with PNT (10\% normal goat serum in PBT) for 30 min at room temperature. Next, samples were incubated with primary antibodies diluted in PNT overnight at $4^{\circ} \mathrm{C}$. Samples were then washed in PBT $(3 \times 15 \mathrm{~min}$ at room temperature), and then incubated overnight in secondary antibody. After being rinsed in PBT for $3 \times 15 \mathrm{~min}$, samples were mounted in Vectashield Fluorescent Mounting Media (Vector Laboratories, Burlingame, USA) and observed. The following antibodies were used: mouse nc82 antibody (1:100, Developmental Studies Hybridoma Bank, University of Iowa, USA), rabbit anti-GFP antibody alexa 488 conjugated (1:400, Invitrogen, USA), goat anti-mouse antibody TRITC-conjugated (1:200, Jackson ImmunoResearch Laboratories, USA).

\subsection{Imaging}

Mounted whole brains were scanned with a confocal microscope (Leica TCS SP5). Stacks of optical sections at $2 \mu \mathrm{m}$ spacing were collected by a Leica $40 \times$ objective lens with $1024 \times 1024$ pixel resolution at $200 \mathrm{~Hz}$. The images were processed with ImageJ (National Institutes of Health, rsbweb.nih.gov/ij/). Figures were prepared using Adobe Photoshop (Adobe System, CA, USA).

\subsection{Statistics}

Student's two-tailed $t$-test was carried out to evaluate the consumption difference between two groups in Figure 1. Two-way ANOVA (analysis of variance) was carried out to analyze variation among and between groups in Figures 2 and 3. One-way ANOVA was carried out to evaluate the consumption difference among multiple groups in Figure 5. Fisher's exact test was used to analyze PER data. Statistical significance was assigned as $* P<0.05, * * P<0.01$, $* * * P<$ $0.001 ;$ n.s., not significant.

\section{Results}

\subsection{Drosophila preference for nutritious food is con- centration-dependent}

To test feeding preference towards sugar directly, we used a modified capillary feeding (CAFE) system as our behavior paradigm. First, several types of sugar (sucrose, fructose, trehalose) solutions were paired with pure water to test whether different foods elicit different levels of attraction. After about $22 \mathrm{~h}$ of starvation, flies showed a significant preference towards these sugars over pure water during a $3 \mathrm{~h}$ test. As shown in Figure 1A and B, when either $100 \mathrm{mmol} \mathrm{L}^{-1}$ sucrose or fructose solution was paired with water, flies
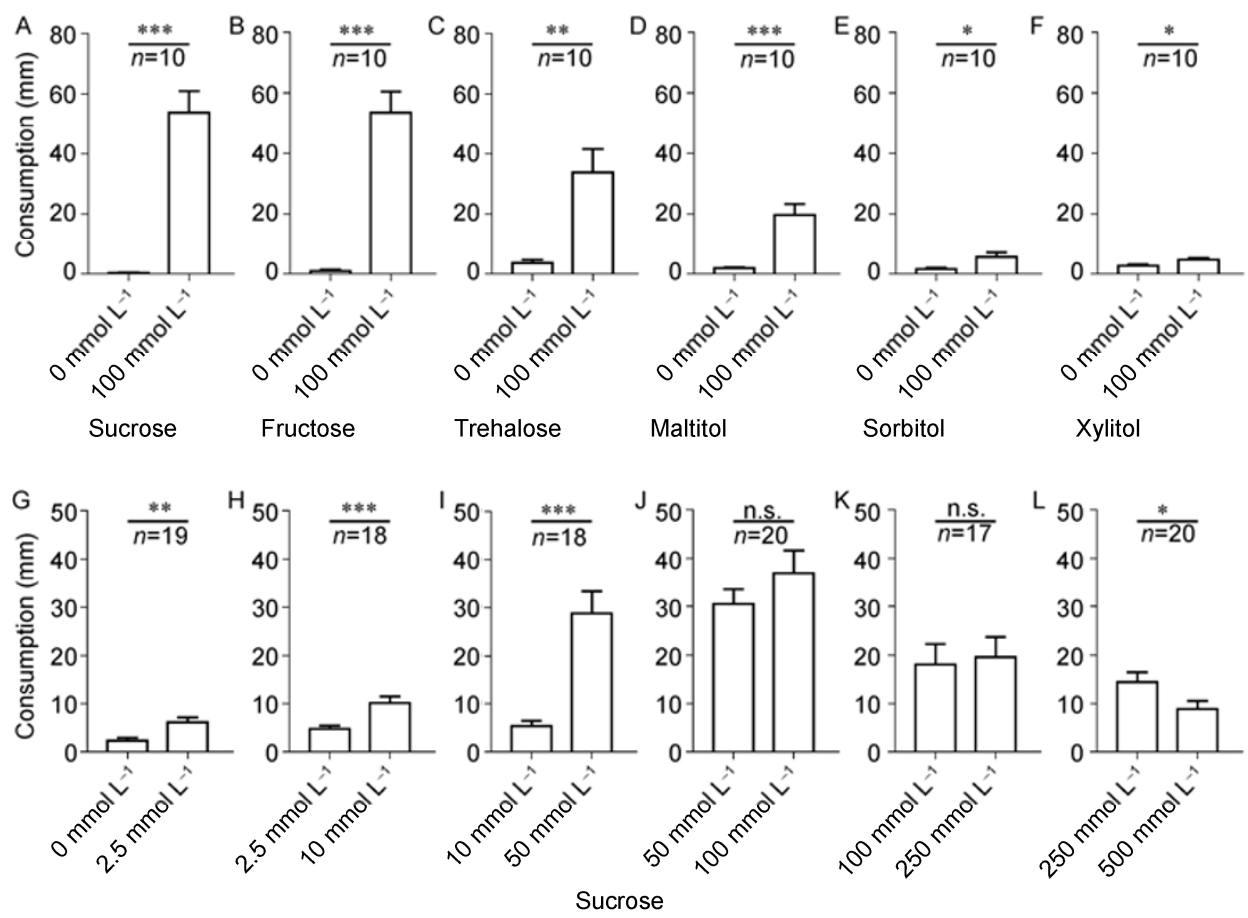

Figure 1 Preference for sweet or nutritious food in Drosophila is concentration dependent. A-F, Wild-type flies were given different foods to test feeding preference. Compared with pure water, flies showed robust preference towards various sweet or nutritious foods, including sucrose (A), fructose (B), trehalose (C), maltitol (D), sorbitol (E) and xylitol (F). G-L, When exposed to a gradient of sucrose concentrations, flies showed sweet preference dimorphism. They were attracted at low and moderate sucrose concentration (G-J), but avoided sucrose at very high concentration (K and L). Error bars indicate SEM. Student's $t$-test, $* P<0.05, * * P<0.01$, $* * * P<0.001$; n.s., not significant. 

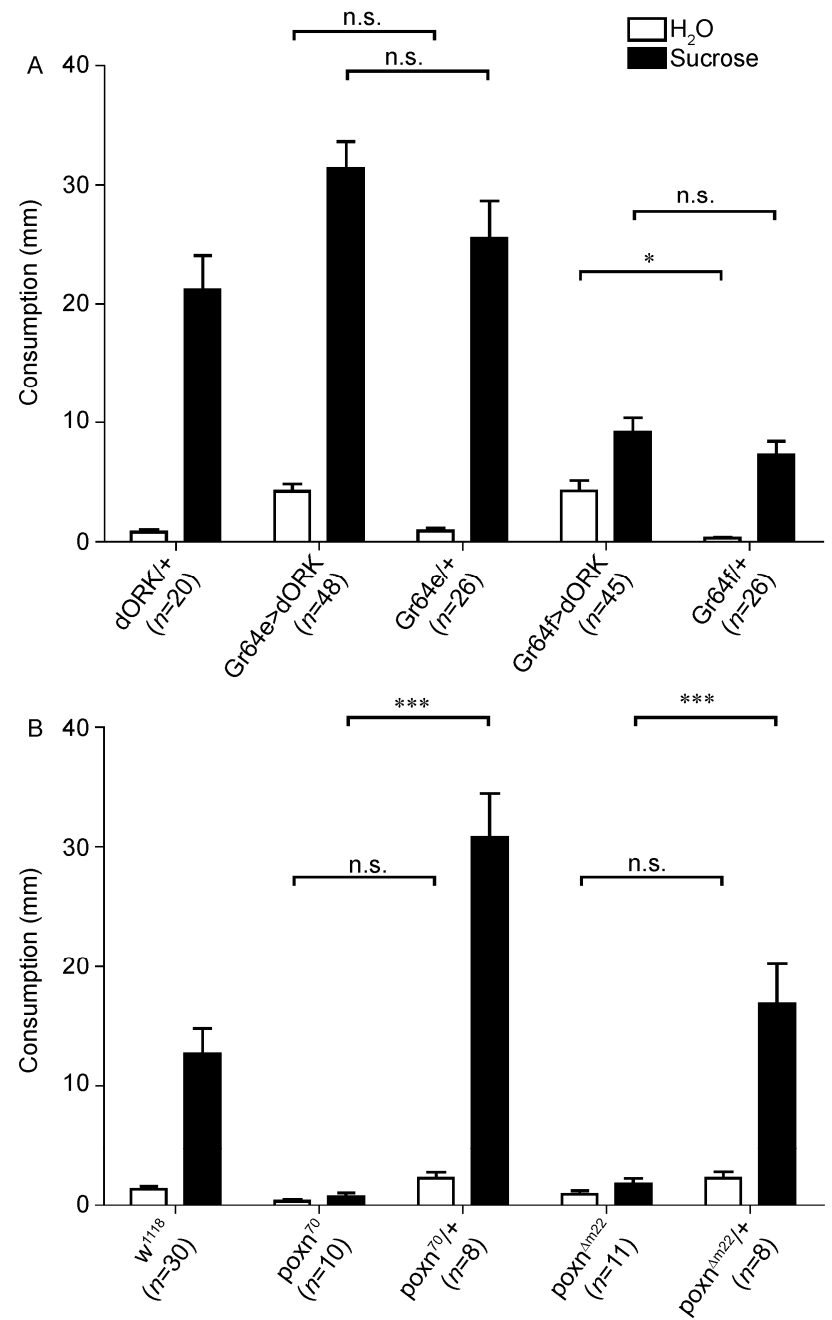

Figure 2 Blocking the taste sensory system decreases sugar preference. Flies with an impaired sugar sensory system showed abnormal feeding behavior. A, After expressing dORK in sugar-sensing neurons driven by Gr64f-Gal4, flies increased water consumption substantially, while sucrose consumption remained unchanged. Expressing dORK in other Gr-Gal4 lines (e.g., Gr64e-Gal4) had little influence on both water and sucrose solution intake. B, poxn null mutants showed a "refusing to eat" phenotype characterized by significantly decreased consumption of sucrose solution but not water. White bars indicate water consumption while black bars indicate sucrose consumption. Error bars indicate SEM. Two-way ANOVA, ${ }^{*} P<0.05, * * P<0.01, * * * P<0.001 ;$ n.s., not significant.

consumed significantly more sugar solution than pure water $(P<0.0001)$, and the total consumption of sugar solution and water was more than $50 \mathrm{~mm}$. When $100 \mathrm{mmol} \mathrm{L}^{-1}$ trehalose solution was paired with water, flies also consumed more trehalose solution than water $(P=0.0012)$, but total consumption decreased down to $35 \mathrm{~mm}$ (Figure 1C).

Several groups have reported that Drosophila is able to evaluate food nutrition independent of taste [9-12]. To address this, we used several types of sugar-alcohol (sorbitol, xylitol, maltitol) solutions, which have nutritional value but sorbitol and xylitol solutions lack sweet taste, to pair with water and test the fly's feeding preference. As shown in Figure 1D, when given water and $100 \mathrm{mmol} \mathrm{L}^{-1}$ maltitol solution (sweet), flies still displayed a robust preference towards maltitol $(P<0.0001)$. However, the total consumption of sugar alcohol solution and water decreased down to $20 \mathrm{~mm}$. Then, we chose to pair two tasteless but energy-rich sugar alcohol, the sorbitol and xylitol solutions, with water respectively. As we expected (Figure $1 \mathrm{E}$ and $\mathrm{F}$ ), the consumption of sorbitol $(P=0.0173)$ or xylitol $(P=0.0388)$ was significantly higher than that of water. However, the total consumption of sugar alcohol solution and water decreased to less than $10 \mathrm{~mm}$. These results indicate that Drosophila always preferred nutritious food over water, regardless of whether it was sweet or not. However, the amount of food intake was different in different food sources.

Although the above experiments showed an obvious inclination towards sugar or sugar alcohol compared with pure water, we found food consumption also varied considerably in a concentration-dependent manner when flies were provided with only sucrose solutions. First, at a low concentration range $\left(0-50 \mathrm{mmol} \mathrm{L}^{-1}\right)$, flies always preferred the "sweeter" food (Figure 1G-I). When given pure water and $2.5 \mathrm{mmol} \mathrm{L}{ }^{-1}$ sucrose solution, flies could discriminate sucrose solution from water $(P=0.0022$, Figure $1 \mathrm{G})$. When given 2.5 and $10 \mathrm{mmol} \mathrm{L}^{-1}$ sucrose solution, flies still showed a significant preference towards the sweeter (10 mmol L $\left.\mathrm{L}^{-1}\right)$ sucrose solution $(P=0.001$, Figure $1 \mathrm{H})$. The preference for sweeter food persisted in the 10 and $50 \mathrm{mmol}$ $\mathrm{L}^{-1}$ sucrose solution group $(P<0.0001$, Figure $1 \mathrm{I})$. When the sucrose concentration increased, the total consumption increased as well. The consumption reached its peak at 50 and $100 \mathrm{mmol} \mathrm{L}^{-1}$ sucrose solution group, however, the consumption of $100 \mathrm{mmol} \mathrm{L}^{-1}$ sucrose solution was not significantly higher than that of $50 \mathrm{mmol} \mathrm{L}^{-1}$ sucrose solution $(P=0.2541$, Figure $1 \mathrm{~J})$. Interestingly, as the sucrose concentration increased even more, the total consumption decreased (Figure $1 \mathrm{~J}-\mathrm{L}$ ). In the 100 and $250 \mathrm{mmol} \mathrm{L}{ }^{-1}$ sucrose solution group, starved flies consumed the two food sources equally $(P=0.8087)$. However, the level of consumption was nearly half of the 50 and $100 \mathrm{mmol} \mathrm{L}^{-1}$ sucrose solution groups (Figure $1 \mathrm{~K}$ ). In the 250 and $500 \mathrm{mmol} \mathrm{L}^{-1}$ sucrose solution groups, the preference was clearly towards the "less sweet" one ( $P=0.0345$, Figure 1L). When we compared solutions having a greater concentration difference (100 mmol L $\mathrm{L}^{-1}$ and $\left.1 \mathrm{~mol} \mathrm{~L}^{-1}\right)$, flies showed a significant preference towards the more moderate concentration. This avoidance of high sugar concentrations was also seen with other sugar solutions, such as trehalose solution (data not shown). These results indicate that Drosophila shows sweet preference dimorphism, attracted by a low and moderate concentration while avoiding a very high concentration.

\subsection{Blocking the taste sensory system eliminates su- crose preference}

To understand the neural circuits responsible for sugar pref- 

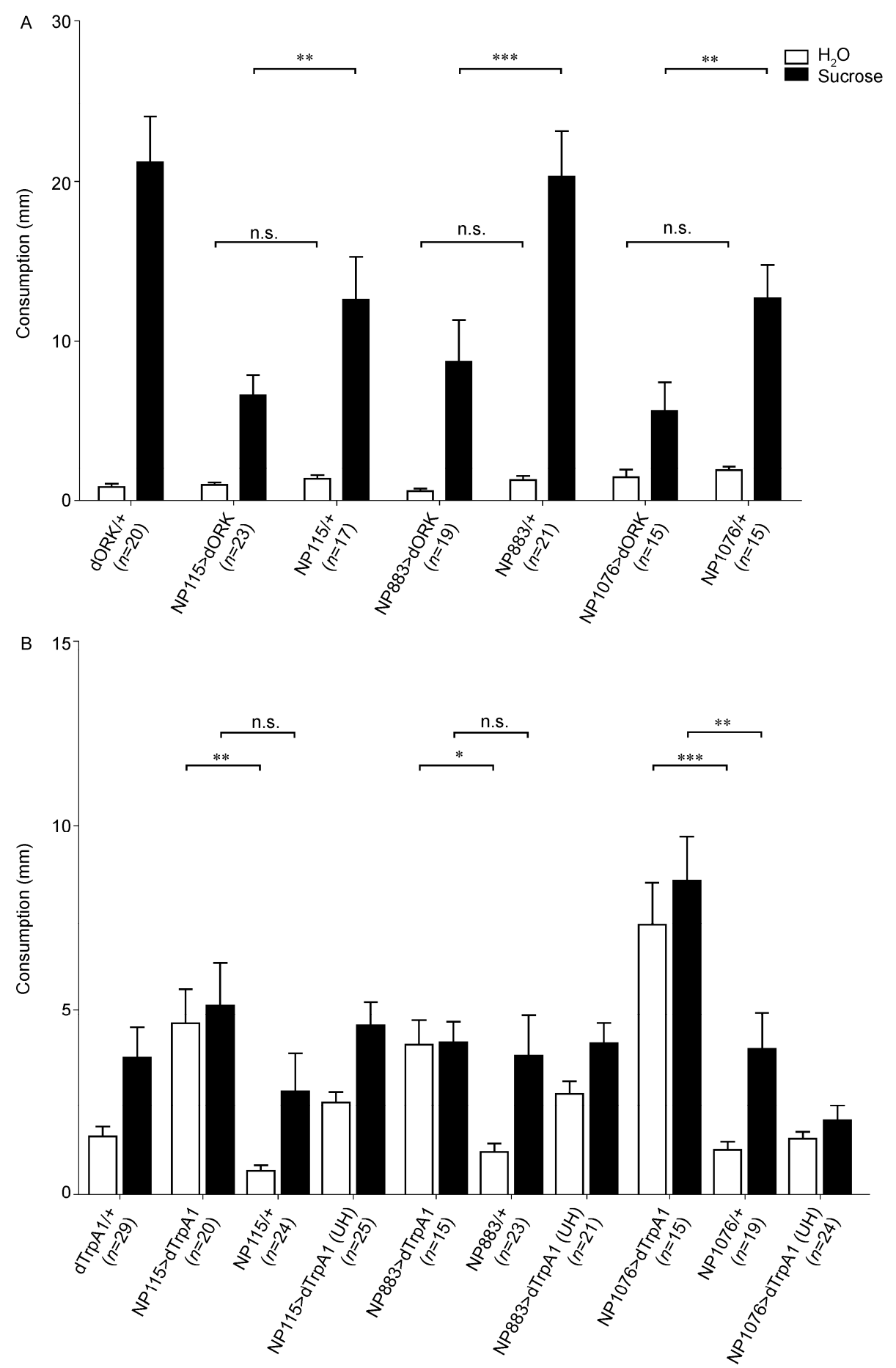

Figure 3 Neural silencing screen identifying three NP-Gal4 labeled circuits involved in feeding behavior. A, When the three Gal4 (NP115-, NP883- and NP1076-Ga14) labeled neurons were silenced, flies decreased their sucrose consumption significantly while maintaining water consumption (A). B, After activation of three NP-Ga14 labeled neurons flies increased water consumption significantly. Only NP1076-Gal4>dTrpA1 activated flies also increased sucrose solution consumption significantly. White bars indicate water consumption while black bars indicate sucrose consumption. Error bars indicate SEM. Two-way ANOVA, $* P<0.05, * * P<0.01, * * * P<0.001 ;$ n.s., not significant.

erence and to identify neurons involved in taste processing, we tested the role of sugar-sensing neurons that were labeled by several Gr-Gal4 (Gr5a-, Gr61a-, Gr64a-, Gr64c-, Gr64d-, Gr64e, Gr64f-Gal4) lines. To silence neuronal activity of these taste receptors, we crossed the Gr-Gal4 lines with UAS-dORK [40], which expresses a mutant $\mathrm{K}^{+}$channel that opens at the resting membrane potential, causing increased $\mathrm{K}^{+}$efflux and therefore membrane hyperpolarization [46]. Flies were provided with water and $10 \mathrm{mmol} \mathrm{L}^{-1}$ sucrose solution (this was the food source used in the fol- 

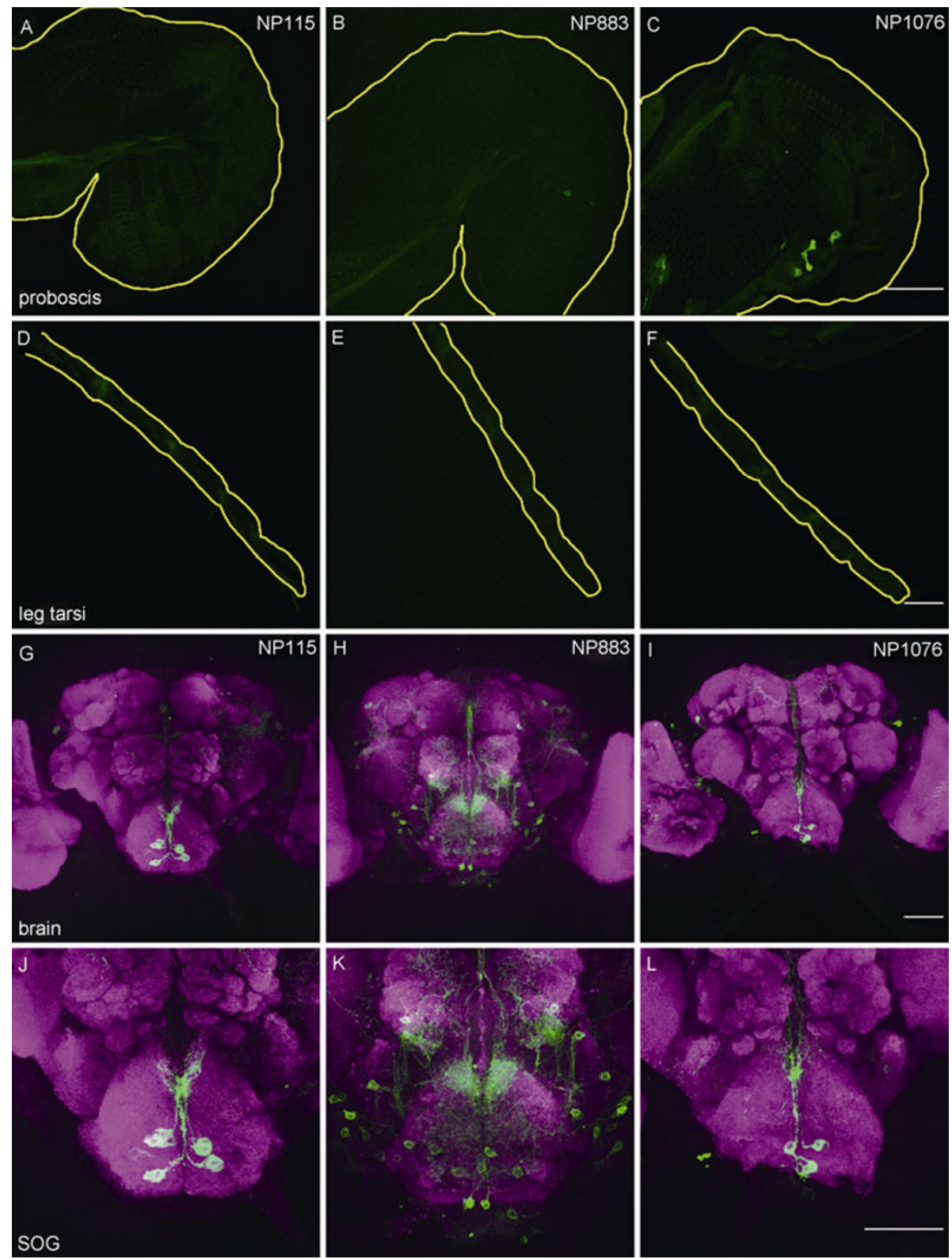

Figure 4 The three NP-Gal4 lines are expressed primarily in the SOG. Membrane tethered GFP was used to report Gal4 expression. A-F, The expression patterns of the three Gal4 lines in periphery sensory organ. No obvious sensory neurons were labeled in either proboscis (A-C) or leg tarsi (D-F). G-L, The expression patterns of the three Gal4 lines in central nervous system. Three Gal4 lines express sparsely across the brain (G-I) but primarily in the SOG (J-L, larger images of G-I SOG region respectively). Green, GFP signal; magenta, nc 82 signal; scale bar, $50 \mu \mathrm{m}$.

lowing experiments unless otherwise noted). Among the seven groups of sugar-sensing neurons that were silenced, only Gr64f-Gal4>UAS-dORK flies decreased their sugar preference, by increasing water consumption substantially. These flies showed increased water consumption up to 4.3 $\mathrm{mm}$, which was significantly higher than controls $(P<0.05)$. However, their sugar consumption was not significantly changed (Figure 2A). Likewise, Gr64e-Gal4>UAS-dORK flies also consumed more water (as much as $4.3 \mathrm{~mm}$ ), which was high but not significant compared with the control group. Similar to Gr64f, the sugar consumption difference between Gr64e-Gal4>UAS-dORK flies and control flies was also not changed (Figure 2A). In contrast, silencing the other five taste receptors revealed normal sugar preference coupled to a small amount of water consumption (less than $3 \mathrm{~mm}$, data not shown). These results showed that neuronal silencing in most of the Gr lines results in a normal sugar preferences, except for one line (Gr64f) showing significantly increased water consumption when given a choice of food sources, which suggests that these sugar-sensing neurons may compensate for each other in sugar detection, and silencing one at a time cannot eliminate sugar preference completely.

To determine whether eliminating a suite of taste receptors might produce a stronger sugar preference defect, we next tested the taste-blind mutants, poxn $^{70}$ and poxn $^{4 M 22}$, in 

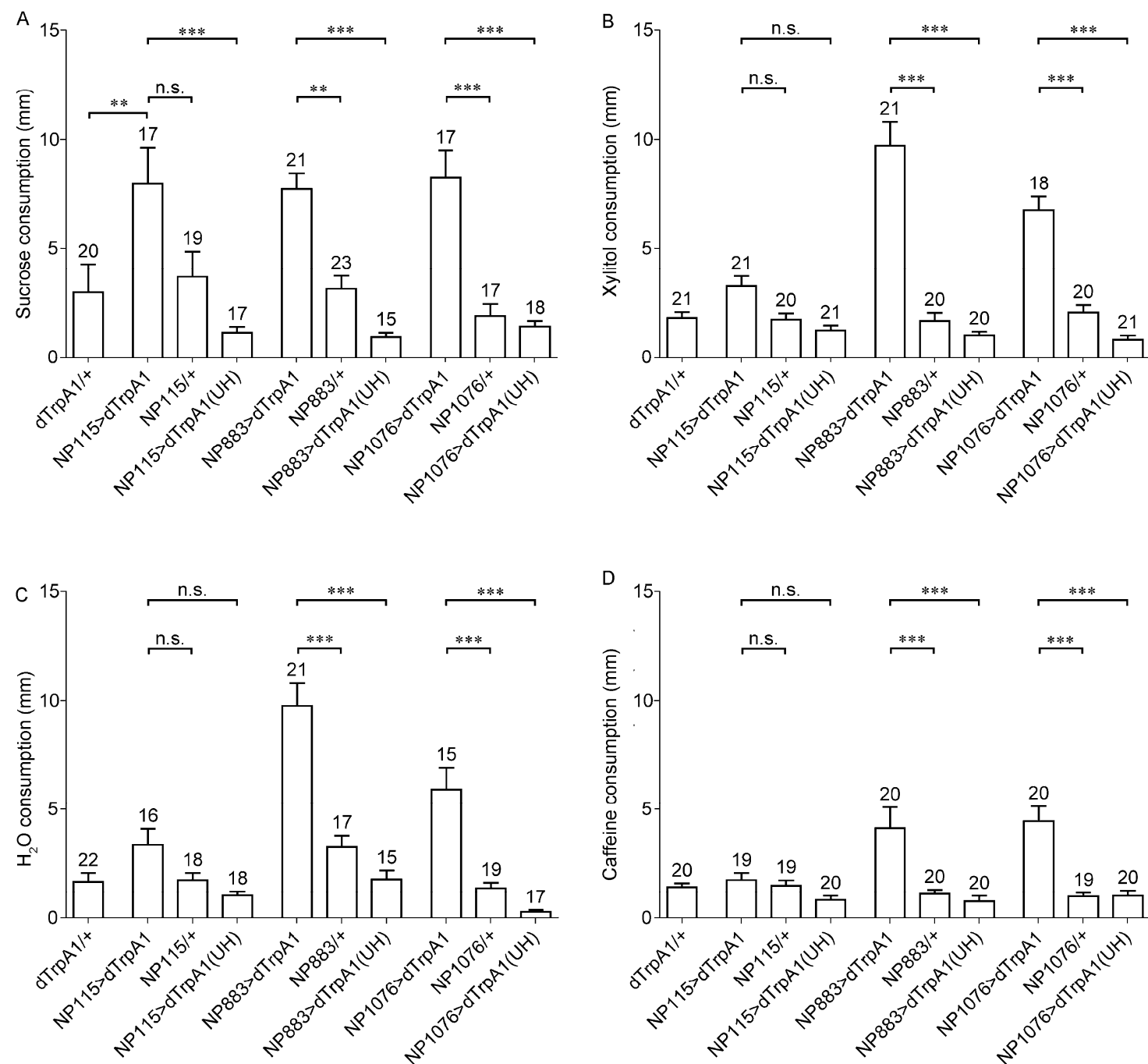

Figure 5 Increasing the activity of the three Gal4 labeled neurons enhanced food intake in different food conditions. Flies were subjected to the CAFE apparatus with only one kind of solution in heated or unheated conditions (UH). The activated NP115-Gal4>UAS-dTrpA1 flies showed increased consumption only in sucrose solution condition, whereas the other two activated lines showed increased consumption in all food conditions, including sucrose solution (A), xylitol solution (B), water (C), caffeine solution (D). Error bars indicate SEM. One-way ANOVA, ${ }^{*} P<0.05, * * P<0.01, * * * P<0.001$; n.s., not significant.

which taste bristles are transformed into mechanosensory bristles lacking gustatory receptors [38,47,48]. As a result of the absence of gustatory attraction to sucrose, poxn mutant flies displayed no preference in the two-choice CAFE assay (Figure 2B), as evident by their dramatically lowered consumption of sucrose solution $(P<0.001)$. In fact, they ate little of either food, showing a "refusal to eat" phenotype. These results indicated that gustatory receptors are indeed needed in normal feeding behavior and blocking the taste sensory system could decrease or even eliminate sugar preference.

\subsection{Three Gal4 lines were identified in a screen for altered feeding behaviors}

To find additional neurons that affect feeding behavior, we screened 456 Gal4 lines crossed with UAS-dORK to silence neurons, and then tested the flies' performance in CAFE system. We found three lines with significantly decreased sucrose consumption when neuronal activity was silenced in these neurons (NP115-, NP883- and NP1076-Gal4; Figure 3A). NP115-Gal4>UAS-dORK flies consumed $6.6 \mathrm{~mm}$ sucrose solution which was significantly less compared with control NP115-Gal4/+ flies $(P<0.01)$. NP883-Gal4>UASdORK flies consumed $8.7 \mathrm{~mm}$ sucrose solution, which was also significantly less compared with control NP883Gal4/+ flies $(P<0.001)$. NP1076-Gal4>UAS-dORK flies consumed $5.6 \mathrm{~mm}$ sucrose solution, which was, again, significantly less than control NP1076-Gal4/+ flies $(P<0.01$, Figure $3 \mathrm{~A})$. Water consumption was not different from controls for all three transgenic lines (Figure 3A). These neural silencing experiments suggest that neurons marked by the 
three NP-Gal4 lines are necessary for sugar intake.

Since silencing these neurons decreased sucrose intake, we questioned whether activating the neurons might have the opposite effect. We monitored the behavioral effect during acute activation of these three Gal4 labeled neurons by using the temperature-inducible activated cation channel dTrpA1 [42]. Interestingly, activating these three circuits primarily increased water consumption, relative to sucrose (Figure 3B). NP115-Gal4>UAS-dTrpA1 activated flies consumed $4.6 \mathrm{~mm}$ of water, which was significantly higher than $0.6 \mathrm{~mm}$ in NP115-Gal4/+ flies $(P<0.01)$. NP883Gal4>UAS-dTrpA1 activated flies consumed $4.0 \mathrm{~mm}$ water, which was also significantly higher than NP883-Gal4/+ flies $(1.1 \mathrm{~mm}, P<0.05)$. Finally, NP1076-Gal4>UASdTrpA1 activated flies consumed as much as $7.3 \mathrm{~mm}$ water, significantly higher compared to the $1.2 \mathrm{~mm}$ water consumption in NP1076-Gal4/+ flies $(P<0.001)$. In contrast, sucrose solution consumption was not elevated to the same degree as that of water. Only NP1076-Gal4>UAS-dTrpA1 activated flies displayed significantly elevated sucrose solution consumption after neuronal activation (Figure 3B). These flies consumed $8.5 \mathrm{~mm}$ sucrose solution, which was significantly higher compared to $3.9 \mathrm{~mm}$ in NP1076-Gal4/+ flies $(P<0.01)$. NP115-Gal4>UAS-dTrpA1 and NP883Gal4>UAS-dTrpA1 activated flies consumed the same amount of sucrose solution as the control groups (Figure 3B). These neuronal activation experiments showed that when the activity of three Gal4 labeled neurons was acutely increased, flies increased liquid consumption significantly, with a preference for water consumption. Taken together, these results indicate that the activity of the three NPGal4 labeled neurons in this study is involved in feeding behavior.

\subsection{The expression pattern of three NP-Gal4 lines was mainly in SOG}

To identify the neurons that are labeled by the three Gal4 lines identified in our screen, we initially used CD8::GFP to characterize the expression pattern of those lines in the peripheral taste organ (proboscis, leg tarsi) as well as in the central nervous system. No obvious GFP signals were observed in peripheral taste organs (Figure 4A-F), which suggested that the neurons in the central nervous system rather than those of the peripheral sensory system were responsible for the food intake phenotypes we uncovered. As shown in Figure 4G-L, GFP signals of three Gal4 lines distribute sparsely across the brain, but intense labeling is seen in SOG, including in cell bodies and neural projections throughout this structure. Although the Gal4 lines had these characteristics in common, they did have some distinct expression patterns. For example, NP115-Gal4 labeled four bundles of neural fibers that converge onto the top of the
SOG, with two localizing in the anterior part and the other two in the posterior part. Within one bundle, more than one cell body was detected. A similar expression pattern was observed in NP1076-Gal4. However, it labeled a few more neurons than NP115-Gal4 line did, including six bundles of fibers that converged together in a pair along the midline, located from anterior to posterior in SOG. NP883-Gal4 showed a more extensive expression pattern compared with the other two Gal4 lines, labeling cell bodies sparsely at the bottom of the SOG, and intense projective arborization at the top of the SOG. These results indicate that among our Gal4 candidates, two label similar neurons while the other one labels a distinct group of neurons (Figure 4J-L). Taken together with our behavioral data, our imaging results suggest that the SOG neurons labeled by these three Gal4 lines might be involved in the regulation of food intake.

\subsection{Increasing the activity of neurons labeled by the three NP-Gal4 lines promotes feeding behavior}

To investigate whether acute neuronal activation of the three Gal4 circuits promotes feeding in general, we provided transgenic flies with a single type of food in the CAFE assay. In this scenario, rather than presenting competing food sources, only a single type of food was presented (10 mmol L ${ }^{-1}$ sucrose solution, $10 \mathrm{mmol} \mathrm{L}^{-1}$ xylitol solution, pure water or $2.5 \mathrm{mmol} \mathrm{L}^{-1}$ caffeine solution). As shown in Figure 5, when fed with $10 \mathrm{mmol} \mathrm{L}^{-1}$ sucrose solution, all three heat-activated flies consumed significantly more sucrose solution than the unheated $(\mathrm{UH})$ controls $(P<0.001$ for each group), as well as the heated parental controls $(P<0.01$; Figure 5A). When fed with $10 \mathrm{mmol} \mathrm{L}^{-1}$ xylitol solution, which is reported as tasteless but nutritious to flies [9], NP883-Gal4>UAS-dTrpA1 flies consumed as much as $9.7 \mathrm{~mm}$ when heated, in comparison to $1.0 \mathrm{~mm}$ when unheated and $1.7 \mathrm{~mm}$ for heated NP883-Gal4/+ control flies $(P<0.001)$. NP1076-Gal4>UAS-dTrpA1 activated flies also significantly enhanced their xylitol solution consumption up to $6.7 \mathrm{~mm}$ compared with two control groups (UH control $0.8 \mathrm{~mm}$, NP1076-Gal4/+ control $2.1 \mathrm{~mm}, P<0.001$; Figure 5B). Only NP115-Gal4>UAS-dTrpA1 activated flies displayed similar xylitol solution consumption $(3.3 \mathrm{~mm})$ as two control groups (1.2 and $1.7 \mathrm{~mm}$, respectively). When flies were fed water, heat activated NP883-Gal4> UAS-dTrpA1 and NP1076-Gal4>UAS-dTrpA1 flies also consumed more: 9.7 and $5.9 \mathrm{~mm}$, respectively, both of which were significantly higher than corresponding controls $(P<0.001)$. However, NP115-Gal4>UAS-dTrpA1 activated flies did not increase their consumption as the other two activated lines (Figure 5C). In addition to these positive or neutral substances, we also tested caffeine, which tastes bitter to flies, as a negative stimulator to test whether flies still increase consumption after activation of three Gal4 
labeled neurons. Interestingly, when fed with $2.5 \mathrm{mmol} \mathrm{L}^{-1}$ caffeine solution, although all the lines consumed less, heat activated NP883-Gal4>UAS-dTrpA1 and NP1076-Gal4> UAS-dTrpA1 flies still consumed more than $4 \mathrm{~mm}$ caffeine solution, which was significantly higher than inactivated controls and heated parental controls $(P<0.001)$. Caffeine consumption in heat activated NP115-Gal4>UAS-dTrpA1 flies was not significantly different from controls (Figure 5D). These results indicate that activation of the neurons labeled by the three Gal4 lines promotes food intake in different manners. The activation of NP115-Gal4 labeled neurons promoted feeding only in food condition of sucrose solution, which is sweet and energy-supportive. The activation of NP883- and NP1076-Gal4 labeled neurons promoted feeding in all four food conditions: sweet and nutritious, tasteless and nutritious, bitter, or neutral.

In the preceding experiments, a food stimulus was always present when we tested feeding behavior. To investigate whether the neurons labeled by the three Gal4 lines affect feeding behavior independent of food stimulation, we measured their proboscis extension reflex (PER) upon transient stimulation, in the absence of a food stimulus. We subjected flies to the PER paradigm by directly activating the Gal4 neurons with temperature sensitive dTrpA1. In the PER assay, the flies, which expressed dTrpA1 driven by the three NP-Gal4 lines, were focally heated with an infrared heat pulse directed to the head. NP883-Gal4>UAS-dTrpA1 flies displayed a $79 \%$ proboscis extension frequency upon application of the laser heat (Figure 6A), which was dramatically enhanced as compared to all the control flies $(P<0.0001$; Figure 6B). Heat activated NP115-Gal4> UAS-dTrpA1 flies (35\%) and NP1076-Gal4>UAS-dTrpA1 flies $(31 \%)$ showed modest but significant enhancement compared with the same flies without heat stimulation or the heated parental controls $(P<0.0001$ except for the comparison of heat activated NP1076-Gal4>UAS-dTrpA1 and NP1076-Gal4/+ flies, $P=0.0032$; Figure 6B). These results showed that induced activation of the three Gal4 labeled neurons can, to some extent, replace the attractive cues represented by foods, which suggests that these neurons might be involved in feeding control as well as motivation to feed.

\section{Discussion}

Various animals show innate attraction to sweet or nutritious food while avoiding bitter or toxic food. This natural feeding behavior is a complex process that depends on both external and internal cues. Understanding how the brain integrates sensory information with internal needs, to direct appropriate feeding behaviors, requires a thorough knowledge of the entire process, from the sensory level to higher-order components in taste circuits. In this study, we have

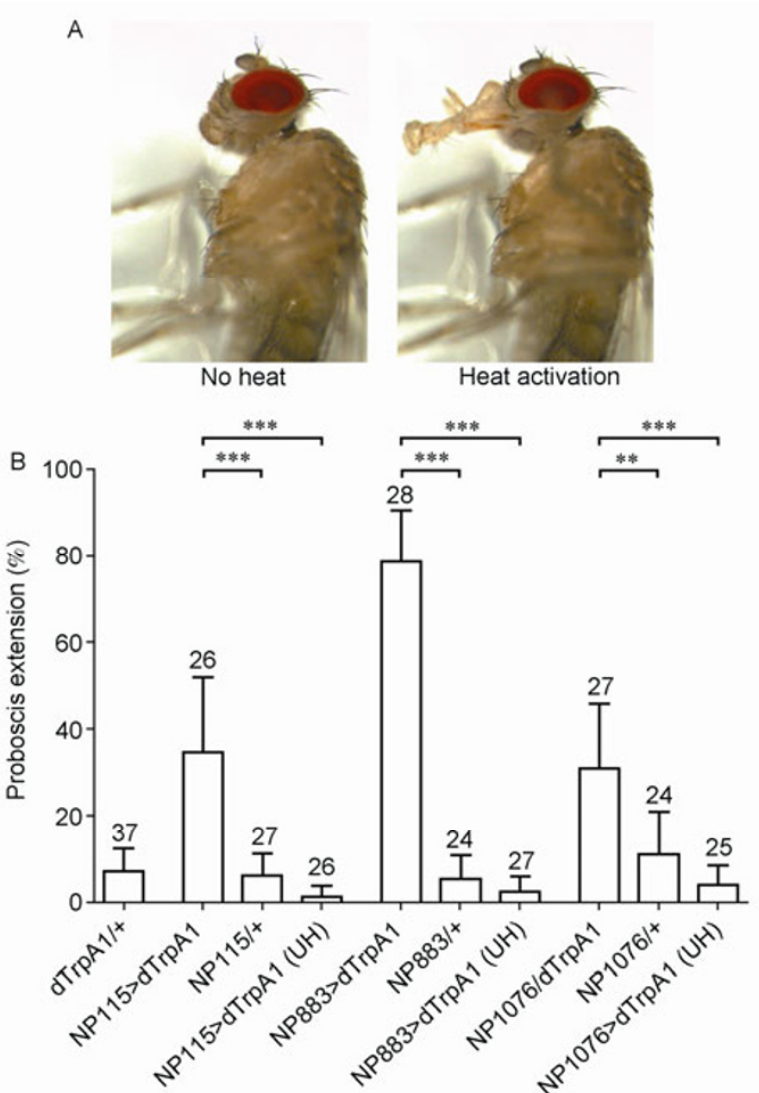

Figure 6 Activation of the three NP-Gal4 labeled neurons promotes feeding independent of food stimulation. A, Example images of NP883-Gal4>UAS-dTrpA1 flies without (left) and with (right) infrared heat stimulation. These flies did not extend their proboscises at room temperature. However, they extended their proboscises once they received a 5 s laser pulse. B, Quantification of PER experiments. All three heat activated flies showed an increased fraction of extension upon heat stimulation, which was not observed in the unheated condition (UH). Error bars indicate $95 \%$ CI (confidence interval). Fisher's exact test, $* * P<0.01$, $* * * P<0.001$.

identified three Gal4 lines (NP115-, NP883- and NP1076Gal4) that label groups of neurons in the central nervous system responsible for feeding behavior. Flies decrease their sugar solution intake when these Gal4 labeled neurons are inhibited, and conversely, enhance their feeding behavior when these neurons are activated, suggesting these neurons might be involved in feeding control.

Because feeding behavior of Drosophila is initiated by stimulation of peripheral sensory organs, flies respond to "sweet" food vigorously by consuming large amounts of it (Figure 1A-D). These "sweet" foods include sugars (sucrose, fructose and trehalose) as well as sugar alcohol (maltitol), which have been shown to elicit electrophysiological and behavioral responses in flies [24]. In comparison, tasteless but energy supportive sugar alcohols (sorbitol and xylitol) $[9,24]$ were consumed much less although the preference over water remained (Figure $1 \mathrm{E}$ and $\mathrm{F}$ ). We surmise 
that at the beginning of the experiment, flies chose randomly between the two kinds of food. The flies provided with water and a sweet food (sugars or maltitol) rapidly recognized the sweet food and demonstrated a strong preference for it. However, those flies provided with water and a metabolizable but tasteless food (sorbitol or xylitol) required time to evaluate the nutritional value by an internal sensor after ingestion [10,12]. Hence, flies in our experimental conditions used both their taste and post-ingestive system to evaluate food sources.

Regarding the consumption discrepancies in the sucrose concentration gradient experiment, flies were indifferent to both lower and higher concentrations, but displayed an obvious preference at intermediate concentrations (Figure $1 \mathrm{G}-\mathrm{L}$ ), which is in agreement with preference of egg-laying site selection on sugars [49]. This behavioral dimorphism, reminiscent of preference towards salt, which is preferred at low and disliked at high concentrations [50-52], might result from the balance between the attractive (palatability, nutrition) and unattractive characteristics (viscosity, stickiness) of the solution [53]. Therefore, in our neural modulating experiments, we mainly used $10 \mathrm{mmol} \mathrm{L}^{-1}$ sucrose solution, which is sweet enough to induce feeding but not too sweet to inhibit feeding.

To establish the criterion for a larger genetic screen, sugar-sensing mutants and Gr mutants were used to test sugar preference. In previous studies, poxn null mutants were found to be completely devoid of sweet sensory input $[38,39]$, whereas the GRN blocking flies may have partially retained the ability to sense sweetness because not all of the sugar sensing neurons were blocked by using one Gr-Gal4 line $[24,30]$. This led to the enhanced water consumption (Figure 2A). Nevertheless, poxn null mutants that lost the motivation to feed barely ingested either solution. This finding, which does not agree with taste-independent nutrition recognition, might be due to a developmental defect of the poxn homozygous mutants we used.

The SOG region is widely regarded as the primary taste center in flies, and receives information from the sensory neurons and sends signals to control feeding movement after local circuit processing $[31,54]$. In recent years, although much progress has been made in identifying first-order sensory neurons and motor neurons controlling feeding movement, a direct connection between the two neuronal populations has not been reported, which suggests that there is at least a one-step relay mediated by interneurons in the SOG [31,32]. Recently one group reported that a single pair of NP883-Gal4 labeled neurons acts as interneurons and directs the feeding motor program [55]. They named this pair of neurons "Fdg neurons" and showed that these neurons are essential for normal feeding behavior through activation and ablation experiments [55]. Their results are consistent with ours in that activation of these neurons triggers
PER and food ingestion. However, we also showed that activation of NP883-Gal4 labeled neurons could enhance food ingestion towards many different solutions other than sugar (Figure 5). The simplest interpretation of our results is that certain neurons labeled by NP883-Gal4 function directly in feeding circuits that are hardwired in the brain. These neurons reside downstream of the first order sensory neurons. On the other hand, activation of these neurons also elicited whole movements required for proboscis extension, which is controlled by a group of motor neurons. Therefore, there is a good likelihood that these candidate neurons labeled by NP883-Gal4 are interneurons, which function as an information relay from sensory neurons to motor neuron. With respect to NP115- and NP1076-Gal4, our loss-offunction and gain-of-function studies argue that although the neurons labeled by these two Gal4 lines also play a role in feeding behavior, their function may be different from NP883-Gal4 labeled neurons. When NP115- and NP1076Gal4 labeled neurons were activated, flies showed a relatively mild PER; their total consumption (especially NP115-Gal4>UAS-dTrpA1 flies) in single type food conditions varied according to food palatability. These results together with the similar expression pattern of NP115- and NP1076-Gal4 lines indicate that those neurons may not be a core part of taste circuits, but might instead be involved in regulating the sensation of starvation or satiety.

To test all of these possibilities and get a deeper understanding of the neural mechanism underlying feeding behavior, further studies are necessary to test and demonstrate directly the functions of the neurons. Furthermore, it will be interesting to narrow down these candidate neurons to more defined neuronal populations, and to determine their specific role in the whole circuit. Overall, our work has identified three groups of brain neurons positively controlling Drosophila feeding behavior, and paves the way for the future investigation of neural mechanism underlying information processing from sensory input to motor output.

We are grateful to Carlson $J R$ (Yale University) for providing the flies. We thank Gong HaiYun and Zhao XuDong of the Institute of Biophysics core facility centre for technical assistance. We also thank the Bloomington Drosophila Stock Center and Drosophila Genetic Resource Center at Kyoto Institute of Technology for fly stocks. This work was supported by the External Cooperation Program of Bureau of International Cooperation, Chinese Academy of Sciences (GJHZ201302), the National Natural Science Foundation of China (31030037), the Ministry of Science and Technology of China (2012CB825504), and the Strategic Priority Research Program B of the Chinese Academy of Sciences (XDB02040200).

1 Gordesky-Gold B, Rivers N, Ahmed OM, Breslin PA. Drosophila melanogaster prefers compounds perceived sweet by humans. Chem Senses, 2008, 33: 301-309

2 Amrein H, Thorne N. Gustatory perception and behavior in Drosophila melanogaster. Curr Biol, 2005, 15: R673-R684

3 Gerber B, Stocker RF. The Drosophila larva as a model for studying 
chemosensation and chemosensory learning: a review. Chem Senses, 2007, 32: 65-89

4 Baker KD, Thummel CS. Diabetic larvae and obese flies - emerging studies of metabolism in Drosophila. Cell Metab, 2007, 6: 257-266

5 Edgecomb RS, Harth CE, Schneiderman AM. Regulation of feeding behavior in adult Drosophila melanogaster varies with feeding regime and nutritional state. J Exp Biol, 1994, 197: 215-235

6 Melcher C, Bader R, Pankratz MJ. Amino acids, taste circuits, and feeding behavior in Drosophila: towards understanding the psychology of feeding in flies and man. J Endocrinol, 2007, 192: 467-472

7 Haselton AT, Fridell YWC. Adult Drosophila melanogaster as a model for the study of glucose homeostasis. Aging, 2008, 2: 523-526

8 Leopold P, Perrimon N. Drosophila and the genetics of the internal milieu. Nature, 2007, 450: 186-188

9 Burke CJ, Waddell S. Remembering nutrient quality of sugar in Drosophila. Curr Biol, 2011, 21: 746-750

10 Dus M, Min S, Keene AC, Lee GY, Suh GS. Taste-independent detection of the caloric content of sugar in Drosophila. Proc Natl Acad Sci USA, 2011, 108: 11644-11649

11 Fujita M, Tanimura T. Drosophila evaluates and learns the nutritional value of sugars. Curr Biol, 2011, 21: 751-755

12 Miyamoto T, Slone J, Song X, Amrein H. A fructose receptor functions as a nutrient sensor in the Drosophila brain. Cell, 2012, 151: 1113-1125

13 Dethier VG. The hungry fly: a physiological study of the behavior associated with feeding. Cambridge: Harvard University Press, 1976

14 Stocker RF. The organization of the chemosensory system in Drosophila melanogaster: a review. Cell Tissue Res, 1994, 275: 3-26

15 Singh RN. Neurobiology of the gustatory systems of Drosophila and some terrestrial insects. Microsc Res Tech, 1997, 39: 547-563

16 Shanbhag SR, Park SK, Pikielny CW, Steinbrecht RA. Gustatory organs of Drosophila melanogaster: fine structure and expression of the putative odorant-binding protein PBPRP2. Cell Tissue Res, 2001, 304: 423-437

17 Ishimoto $\mathrm{H}$, Tanimura T. Molecular neurophysiology of taste in Drosophila. Cell Mol Life Sci, 2004, 61: 10-18

18 Wang Z, Singhvi A, Kong P, Scott K. Taste representations in the Drosophila brain. Cell, 2004, 117: 981-991

19 Clyne PJ, Warr CG, Carlson JR. Candidate taste receptors in Drosophila. Science, 2000, 287: 1830-1834

20 Dunipace L, Meister S, McNealy C, Amrein H. Spatially restricted expression of candidate taste receptors in the Drosophila gustatory system. Curr Biol, 2001, 11: 822-835

21 Scott K, Brady R Jr., Cravchik A, Morozov P, Rzhetsky A, Zuker C, Axel R. A chemosensory gene family encoding candidate gustatory and olfactory receptors in Drosophila. Cell, 2001, 104: 661-673

22 Robertson HM, Warr CG, Carlson JR. Molecular evolution of the insect chemoreceptor gene superfamily in Drosophila melanogaster. Proc Natl Acad Sci USA, 2003, 100(Suppl 2): 14537-14542

23 Thorne N, Chromey C, Bray S, Amrein H. Taste perception and coding in Drosophila. Curr Biol, 2004, 14: 1065-1079

24 Dahanukar A, Lei YT, Kwon JY, Carlson JR. Two Gr genes underlie sugar reception in Drosophila. Neuron, 2007, 56: 503-516

25 Jiao Y, Moon SJ, Wang X, Ren Q, Montell C. Gr64f is required in combination with other gustatory receptors for sugar detection in Drosophila. Curr Biol, 2008, 18: 1797-1801

26 Slone J, Daniels J, Amrein H. Sugar receptors in Drosophila. Curr Biol, 2007, 17: 1809-1816

27 Dahanukar A, Foster K, van der Goes van Naters WM, Carlson JR. A Gr receptor is required for response to the sugar trehalose in taste neurons of Drosophila. Nat Neurosci, 2001, 4: 1182-1186

28 Ueno K, Ohta M, Morita H, Mikuni Y, Nakajima S, Yamamoto K, Isono K. Trehalose sensitivity in Drosophila correlates with mutations in and expression of the gustatory receptor gene Gr5a. Curr Biol, 2001, 11: 1451-1455
29 Jiao Y, Moon SJ, Montell C. A Drosophila gustatory receptor required for the responses to sucrose, glucose, and maltose identified by mRNA tagging. Proc Natl Acad Sci USA, 2007, 104: 14110-14115

30 Wisotsky Z, Medina A, Freeman E, Dahanukar A. Evolutionary differences in food preference rely on Gr64e, a receptor for glycerol. Nat Neurosci, 2011, 14: 1534-1541

31 Gordon MD, Scott K. Motor control in a Drosophila taste circuit. Neuron, 2009, 61: 373-384

32 Manzo A, Silies M, Gohl DM, Scott K. Motor neurons controlling fluid ingestion in Drosophila. Proc Natl Acad Sci USA, 2012, 109: 6307-6312

33 Inagaki HK, Ben-Tabou de-Leon S, Wong AM, Jagadish S, Ishimoto H, Barnea G, Kitamoto T, Axel R, Anderson DJ. Visualizing neuromodulation in vivo: TANGO-mapping of dopamine signaling reveals appetite control of sugar sensing. Cell, 2012, 148: 583-595

34 Marella S, Mann K, Scott K. Dopaminergic modulation of sucrose acceptance behavior in Drosophila. Neuron, 2012, 73: 941-950

35 Itskov PM, Ribeiro C. The dilemmas of the gourmet fly: the molecular and neuronal mechanisms of feeding and nutrient decision making in Drosophila. Front Neurosci, 2013, 7: 12

36 Yarmolinsky DA, Zuker CS, Ryba NJP. Common sense about taste: from mammals to insects. Cell, 2009, 139: 234-244

37 Guo A, Li L, Xia SZ, Feng CH, Wolf R, Heisenberg M. Conditioned visual flight orientation in Drosophila: dependence on age, practice, and diet. Learn Mem, 1996, 3: 49-59

38 Awasaki T, Kimura K. pox-neuro is required for development of chemosensory bristles in Drosophila. J Neurobiol, 1997, 32: 707-721

39 Boll W, Noll M. The Drosophila Pox neuro gene: control of male courtship behavior and fertility as revealed by a complete dissection of all enhancers. Development, 2002, 129: 5667-5681

40 Nitabach MN, Blau J, Holmes TC. Electrical silencing of Drosophila pacemaker neurons stops the free-running circadian clock. Cell, 2002, 109: 485-495

41 Weiss LA, Dahanukar A, Kwon JY, Banerjee D, Carlson JR. The molecular and cellular basis of bitter taste in Drosophila. Neuron, 2011, 69: 258-272

42 Hamada FN, Rosenzweig M, Kang K, Pulver SR, Ghezzi A, Jegla TJ, Garrity PA. An internal thermal sensor controlling temperature preference in Drosophila. Nature, 2008, 454: 217-220

43 Lee T, Luo L. Mosaic analysis with a repressible cell marker for studies of gene function in neuronal morphogenesis. Neuron, 1999, 22: 451-461

44 Ja WW, Carvalho GB, Mak EM, de la Rosa NN, Fang AY, Liong JC, Brummel T, Benzer S. Prandiology of Drosophila and the CAFE assay. Proc Natl Acad Sci USA, 2007, 104: 8253-8256

45 Pan YF, Zhou YQ, Guo C, Gong HY, Gong ZF, Liu L. Differential roles of the fan-shaped body and the ellipsoid body in Drosophila visual pattern memory. Learn Mem, 2009, 16: 289-295

46 Hodge JJ. Ion channels to inactivate neurons in Drosophila. Front Mol Neurosci, 2009, 2: 13

47 Dambly-Chaudiere C, Jamet E, Burri M, Bopp D, Basler K, Hafen E, Dumont N, Spielmann P, Ghysen A, Noll M. The paired box gene pox neuro: a determinant of poly-innervated sense organs in Drosophila. Cell, 1992, 69: 159-172

48 Nottebohm E, Usui A, Therianos S, Kimura K, Dambly-Chaudiere C, Ghysen A. The gene poxn controls different steps of the formation of chemosensory organs in Drosophila. Neuron, 1994, 12: 25-34

49 Wang YJ, Wen SY, Gong HY, Gong ZF, Liu L. A model system for analyzing behavioral preference and plasticity in Drosophila egg-laying. Prog Biochem Biophys, 2012, 39: 910-918

50 Nakamura M, Baldwin D, Hannaford S, Palka J, Montell C. Defective proboscis extension response (DPR), a member of the Ig superfamily required for the gustatory response to salt. J Neurosci, 2002, 22: $3463-3472$ 
51 Liu L, Leonard AS, Motto DG, Feller MA, Price MP, Johnson WA, Welsh MJ. Contribution of Drosophila DEG/ENaC genes to salt taste. Neuron, 2003, 39: 133-146

52 Zhang YV, Ni J, Montell C. The molecular basis for attractive salt-taste coding in Drosophila. Science, 2013, 340: 1334-1338

53 Schipanski A, Yarali A, Niewalda T, Gerber B. Behavioral analyses of sugar processing in choice, feeding, and learning in larval Dro- sophila. Chem Senses, 2008, 33: 563-573

54 Miyazaki T, Ito K. Neural architecture of the primary gustatory center of Drosophila melanogaster visualized with GAL4 and LexA enhancer-trap systems. J Comp Neurol, 2010, 518: 4147-4181

55 Flood TF, Iguchi S, Gorczyca M, White B, Ito K, Yoshihara M. A single pair of interneurons commands the Drosophila feeding motor program. Nature, 2013, 499: 83-87

Open Access This article is distributed under the terms of the Creative Commons Attribution License which permits any use, distribution, and reproduction in any medium, provided the original author(s) and source are credited. 